University of Nebraska - Lincoln

DigitalCommons@University of Nebraska - Lincoln

\title{
Mapping Surface Polarization in Thin Films of the Ferroelectric Polymer P(VDF-TrFE).
}

\author{
Bradley W. Peterson \\ University of Nebraska-Lincoln, bradley.peterson@uwc.edu \\ Stephen Ducharme \\ University of Nebraska, sducharme1@unl.edu \\ Vladimir M. Fridkin \\ Institute of Crystallography, Russian Academy of Sciences, Moscow, fridkin@ns.crys.ras.ru \\ Timothy J. Reece \\ University of Nebraska-Lincoln, reecetj@unk.edu
}

Follow this and additional works at: https://digitalcommons.unl.edu/physicsducharme

Part of the Condensed Matter Physics Commons, and the Polymer and Organic Materials Commons

Peterson, Bradley W.; Ducharme, Stephen; Fridkin, Vladimir M.; and Reece, Timothy J., "Mapping Surface Polarization in Thin Films of the Ferroelectric Polymer P(VDF-TrFE)." (2004). Stephen Ducharme Publications. 61.

https://digitalcommons.unl.edu/physicsducharme/61

This Article is brought to you for free and open access by the Research Papers in Physics and Astronomy at DigitalCommons@University of Nebraska - Lincoln. It has been accepted for inclusion in Stephen Ducharme Publications by an authorized administrator of DigitalCommons@University of Nebraska - Lincoln. 


\title{
Mapping Surface Polarization in Thin Films of the Ferroelectric Polymer P(VDF-TrFE)
}

\author{
B. W. PETERSON

\section{STEPHEN DUCHARME*}

\author{
Department of Physics and Astronomy \\ Center for Materials Research and Analysis \\ University of Nebraska
}

\section{M. FRIDKIN}

Department of Physics and Astronomy Center for Materials Research and Analysis University of Nebraska and Institute of Crystallography Russian Academy of Sciences

Moscow, Russia

\section{TIMOTHY J. REECE}

Department of Physics and Astronomy Center for Materials Research and Analysis University of Nebraska

Received August 8, 2003; in final form January 5, 2004

Pyroelectric Scanning Microscopy (PSM) has been developed to enable mapping of surface polarization in ferroelectric thin films, in particular the copolymer polyvinylidene fuoride triflourorethylene, or $P(V D F-T r F E)$. The Chynoweth method for dynamically measuring pyroelectric current is employed in conjunction with a micropositioning system to construct two-dimensional images of the film polarization. These images have revealed an enhancement of the polarization near the edges of the film below the average coercive field, with the center's polarization increasing thereafter to meet the edge value at saturation.

Keywords Ferroelectricity; vinylidene fluoride; polymer; Langmuir-Blodgett; pyroelectric

PACS: 77.70.+a; 77.80.Dj; 77.84.Jd

\section{Material and LB Films}

The material under study is a copolymer of vinylidene fluoride and trifluoroethylene, $\mathrm{P}(\mathrm{VDF}-\mathrm{TrFE})$, in a 70:30 ratio. In the ferroelectric phase, the polymer chains exist in an all-trans conformation, with successive carbon atoms alternately bonded to fluorine and hydrogen atoms and arranged to produce a net polarization [1].

Address correspondence to B. W. Peterson, Department of Physics and Astronomy, Center for Materials Research and Analysis, University of Nebraska, Lincoln, NE 68588-0111, USA. E-mail: bpeter15@bigred.unl.edu

*E-mail: sducharmel@unl,edu 


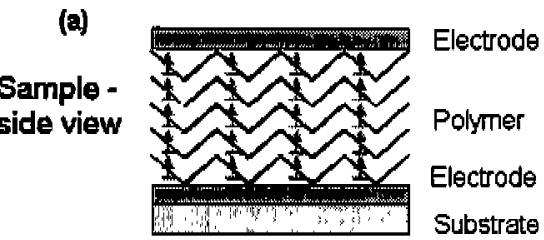

(b)
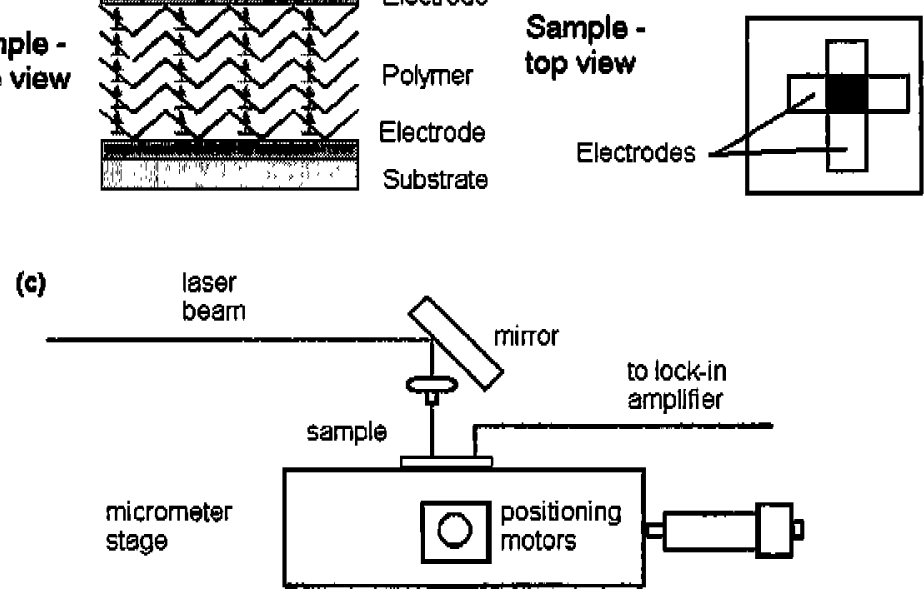

FIGURE 1 (a) A side view of the sample shows the electrodes with the LB film between them, forming a capacitor. (b) A top view shows the cross-section scanned using the PSM technique. (c) A side view of the PSM apparatus.

Samples were prepared as ultra-thin films using the Langmuir-Blodgett (LB) deposition technique [2]. An aluminum electrode was evaporated onto the surface of a glass substrate. The polymer was dispersed on the surface of an ultra-pure water bath, forming a single molecular layer on the surface. As the substrate was dipped into the surface, monolayers (MLs) of the polymer are deposited one at a time. When the sample reached the desired thickness, another aluminum electrode was evaporated onto the sample perpendicular to the first, forming a capacitor (Fig. 1a and b).

\section{Pyroelectric Scanning Microscopy}

Pyroelectric Scanning Microscopy (PSM) was developed from the Chynoweth method for dynamic measurements of pyroelectric currents [3]. A pyroelectric current is generated with a laser beam modulated by an optical chopper operating at fixed frequency, so that the signal is proportional to the sample polarization in the area heated by the laser.

Normally, the Chynoweth measurements are made with an unfocused laser beam, illuminating the entire sample (Fig. 1). We obtain the spatial distribution of polarization by focusing the laser beam and scanning it over the sample. The spatial resolution is limited by the largest of three factors: the beam diameter, the thermal diffusion length, or the scanning step size. The beam diameter depends on the wavelength and microscope objective, while the thermal diffusion length depends on the thermal conductivity of the substrate and the laser modulation frequency.

Our PSM apparatus uses an $8 \mathrm{~mW}$ He-Ne laser modulated at $2 \mathrm{kHz}$ by a chopper, and focused by a microscope objective to illuminate the sample. A micrometer table holds the sample for scanning. Prior PSM studies of the ferroelectric copolymer LB films demonstrated the ability of this technique to make movies of switching $[4,5]$ and to make detailed images of the polarization distribution [2].

\section{Results}

One of the unexpected discoveries made with PSM was the observation of an edge enhancement effect. For applied fields less than the coercive field, it was found that one set of a 


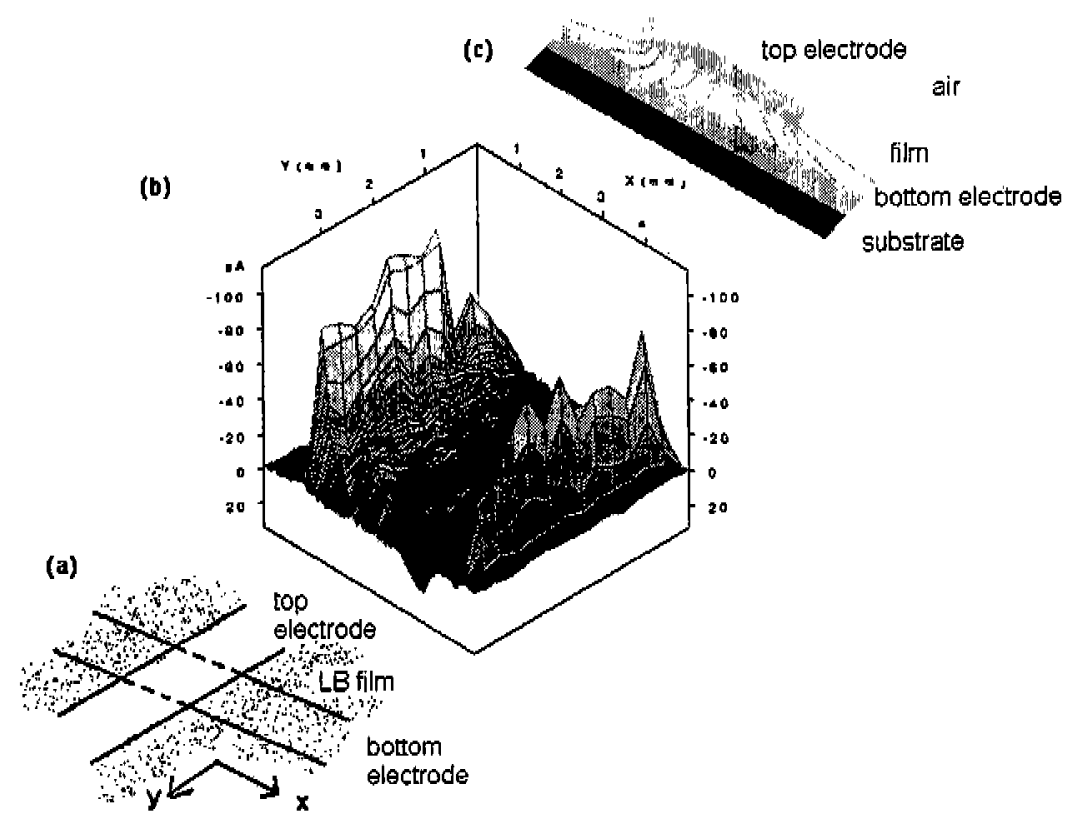

FIGURE 2 (a) The LB film covers the bottom electrode, but not the top. The electrodes have the same orientation as in (b), which shows a surface polarization map made with PSM. (c) The edge enhancement results from the high density of electric field lines at the edge of the top electrode.

sample's edges polarized well before the rest of the sample (Fig. 2b). As the applied field increased, the polarization at the center of the sample rose to meet the edges near saturation.

Figure 3 represents part of an edge enhancement study. Prior to the $-15 \mathrm{~V}$ polarization where the figure begins, the sample was annealed, eliminating the net polarization, then

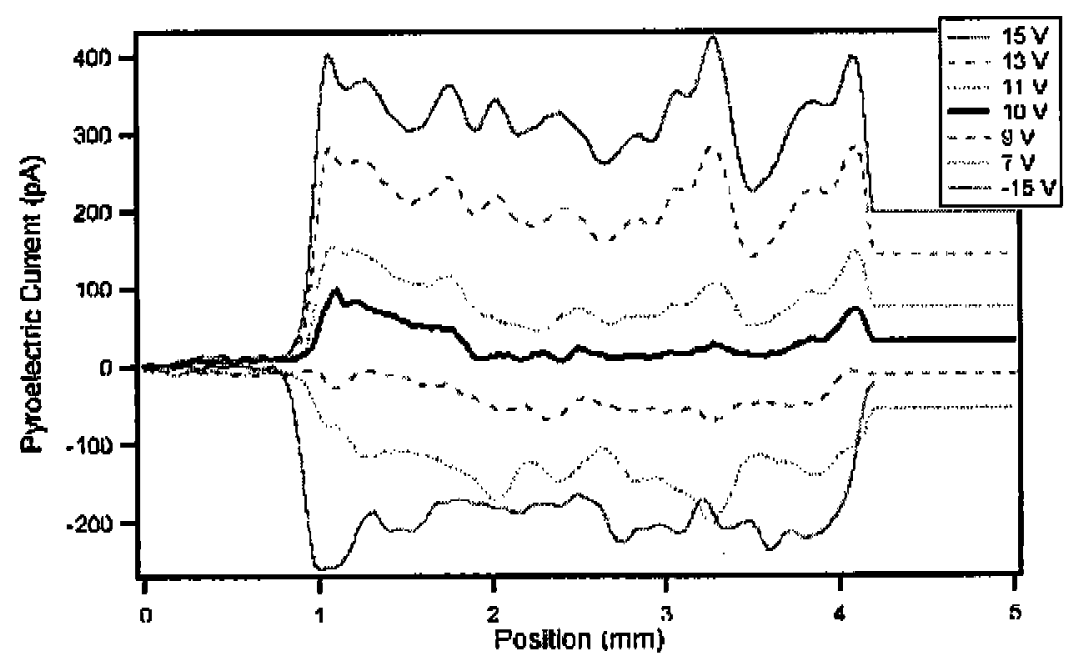

FIGURE 3 A part of a hysteresis loop, from $-15 \mathrm{~V}$ to $15 \mathrm{~V}$ applied voltage, is shown. Data was taken by scanning across a single line of the sample. The coercive voltage, at $10 \mathrm{~V}$, is highlighted, and shows prominent edge enhancement. 
polarized at zero to $15 \mathrm{~V}$ and from $15 \mathrm{~V}$ to $-15 \mathrm{~V}$ in $1 \mathrm{~V}$ increments. The figure contains data from the $-15 \mathrm{~V}$ to $+15 \mathrm{~V}$ section of the loop. A single line was scanned after each voltage was applied. At the coercive field of $+10 \mathrm{~V}$, the edges of the sample show significant polarization, while the center is barely polarized: At higher voltages, the center reaches roughly $75 \%$ of the polarization at the edges. Repetitions of several hysteresis cycles have shown similar results. This particular sample was not fully saturated at $\pm 15 \mathrm{~V}$; other fully saturated samples have shown relatively uniform polarization across their entire surfaces.

The edge enhancement effect was also studied in terms of the orientation of the polymer chains and electrodes. Two samples were fabricated: one with the polymer chains predominantly parallel to the bottom electrode and the other with the chains predominantly perpendicular to the bottom electrode. The edge enhancement appeared in both samples, though less clearly in the latter, possibly because of inhomogeneities in the sample. In both samples, the strongly polarized edges ran parallel to the top electrode (and perpendicular to the bottom).

The edge enhancement effect can be understood by considering the sample as a capacitor, where the bottom electrode is an infinite plane and the top electrode is finite, as sketched in Fig. 2c. The electric field lines become denser near the electrode edges. This explains why the edges polarize more quickly than the rest of the sample. The preference for the top electrode edges could be due to the inherent asymmetry in the edge shapes, the contouring of the polymer film, and the glass versus air interface.

\section{Conclusions}

As interest in ferroelectric thin films for commercial applications increases, it becomes increasingly necessary to have suitable diagnostic techniques to ensure quality control. PSM has proven capable of finding variations in the surface polarization. Already, a significant and regularly occurring inhomogeneity, edge enhancement, has been observed and characterized. While this effect involved the LB film less than the electrodes, flaws in the film itself can also be detected.

\section{Acknowledgements}

We thank John Beezley for help with the electric field modeling. This work was supported by the USA National Science Foundation, the USA Office of Naval Research, the Nebraska Research Initiative, and the Undergraduate Creative and Research Experience fund. Work at the Institute of Crystallography was supported by the Russian Foundation for Basic Research.

\section{References}

1. L. M. Blinov, V. M. Fridkin, S. P. Palto, A. V. Bune, P. A. Dowben, and S. Ducharme, Physics Upsekhi 43, 243 (2000).

2. S. Ducharme, S. P. Palto, and V. M. Fridkin, Ferroelectric polymer langmuir-blodgett films, ch. 11 in Thin Films Handbook, vol. 3: Ferroelectric and Dielectric Thin Films (H. S. Nalwa, ed.) Academic Press, San Diego (2002).

3. A. G. Chynoweth, J. Appl, Phys. 27, 78 (1956).

4. C. Bacon, Profile of a Ferroelectric Polymer, Final Report, Research Experiences for Undergraduates in Nanostructured Research, University of Nebraska (1998).

5. S. Ducharme, M. Bai, M. Poulsen, S. Adenwalla, S. P. Palto, L. M. Blinov, and V. M. Fridkin, Ferroelectrics 252, 191 (2001). 